\title{
A pilot study to understand feasibility and acceptability of stool and cord blood sample collection for a large-scale longitudinal birth cohort
}

S. R. Bailey ${ }^{1}$, C. L. Townsend ${ }^{1}$, H. Dent ${ }^{2}$, C. Mallet ${ }^{2}$, E. Tsaliki ${ }^{3}$, E. M. Riley ${ }^{4}$, M. Noursadeghi ${ }^{3}$, T. D. Lawley ${ }^{5}$, A. J. Rodger ${ }^{6}$, P. Brocklehurst ${ }^{7,8}$ and N. Field ${ }^{6 *}$

\begin{abstract}
Background: Few data are available to guide biological sample collection around the time of birth for large-scale birth cohorts. We are designing a large UK birth cohort to investigate the role of infection and the developing immune system in determining future health and disease. We undertook a pilot to develop methodology for the main study, gain practical experience of collecting samples, and understand the acceptability of sample collection to women in late pregnancy.
\end{abstract}

Methods: Between February-July 2014, we piloted the feasibility and acceptability of collecting maternal stool, baby stool and cord blood samples from participants recruited at prolonged pregnancy and planned pre-labour caesarean section clinics at University College London Hospital. Participating women were asked to complete acceptability questionnaires.

Results: Overall, 265 women were approached and 171 (65\%) participated, with $\geq 1$ sample collected from 113 women or their baby (66\%). Women had a mean age of 34 years, were primarily of white ethnicity $(130 / 166,78 \%)$, and half were nulliparous $(86 / 169,51 \%)$. Women undergoing planned pre-labour caesarean section were more likely than those who delivered vaginally to provide $\geq 1$ sample ( $98 \%$ vs $54 \%$ ), but less likely to provide maternal stool (10\% vs 43\%). Pre-sample questionnaires were completed by 110/171 women (64\%). Most women reported feeling comfortable with samples being collected from their baby ( $<10 \%$ uncomfortable), but were less comfortable about their own stool (19\% uncomfortable) or a vaginal swab (24\% uncomfortable).

Conclusions: It is possible to collect a range of biological samples from women around the time of delivery, and this was acceptable for most women. These data inform study design and protocol development for large-scale birth cohorts.

Keywords: Biological samples, Infant faeces, Cord blood, Feasibility, Acceptability, Large-scale birth cohorts, Bioarchive

\footnotetext{
* Correspondence: nigel.field@ucl.ac.uk

${ }^{6}$ Institute for Global Health, University College London, London, UK

Full list of author information is available at the end of the article
} 


\section{Background}

Large-scale cohort studies increasingly collect biological samples which, combined with survey data, might provide valuable insights into the biological mechanisms of disease [1-4]. Storage of samples in large long-term bioarchives has become common, with the aim of using both current and anticipated future molecular technologies to analyse the samples $[5,6]$. Reductions in costs of molecular testing, in particular for genomics, proteomics and metabolomics applications, have made such approaches attractive to public health researchers $[2,7]$. However, collecting such samples poses multiple challenges, including how to ensure that the proportion of participants providing samples is sufficiently high, and practical challenges associated with working in clinical settings where medical considerations often take priority over research $[8,9]$.

We are designing a new large-scale UK birth cohort study to investigate how exposure to microorganisms and immunological events during pregnancy and early life influence health and disease outcomes across the life course [10].

We undertook the experimental pilot study described here to: (1) inform decisions about methodology for the main study, (2) develop experience about the practicalities and logistical challenges of collecting samples, and (3) gain understanding of the acceptability of sample collection to women in the late stages of pregnancy and around the time of delivery.

\section{Methods}

\section{Study design}

We aimed to collect samples from approximately 100 women around the time of delivery at University College London Hospital (UCLH). Women were recruited between 17th February 2014 and 4th July 2014 by two research midwives, working normal office hours ( 9 am to $5 \mathrm{pm})$. All pregnant women with singleton pregnancies attending either a prolonged pregnancy clinic (gestation $41^{+5}$ or $^{6}$ ) or a planned pre-labour caesarean section clinic up to a week prior to expected delivery were eligible. We selected these groups as containing women who attend outpatient appointments and who might therefore be approached by study midwives in the week prior to labour to discuss the study. Women with multiple pregnancies, those who needed a translator to consent to participate, those under 16 years of age and non-UK residents who intended to return abroad immediately after delivery were excluded.

We aimed for a sample size of 100 participants to estimate with reasonable precision the proportion returning each of the sample types. The study was not designed or powered to detect differences by subgroups.

\section{Sample collection}

Cord blood and stool were chosen for collection as these samples were subject to the greatest uncertainty regarding the optimum processes required for collection, as well as acceptability to women. Study protocols developed as a result of this pilot study are available in Additional file 1.

Cord blood was collected in the maternity unit soon after delivery by a research midwife or by the attending midwife if the delivery occurred outside normal office hours. Cord blood banking was available at UCLH for those women who wanted to donate cord blood and this service was given precedence where women agreed to both.

Women were asked to provide a stool sample in the maternity unit before or after delivery, or stool was collected during delivery by midwives. The first baby stool sample (meconium) was collected while the baby remained in hospital. For a subset of ten women recruited in May and June 2014, an additional baby stool collection protocol was established and tested for home collection of stool every other day after birth: samples were taken by women and collected from their home by courier. These samples were used for experimental validation of our procedures. After home collection of baby stool commenced, women not enrolled in home collection were only asked to consent to collection of cord blood and maternal stool. Home collection of baby stool ended on 9th June 2014, after which all collection of maternal stool and baby stool collection stopped; only cord blood was collected from this date until the end of the study (4th July 2014). We were not able to collect reasons for failing to get a sample.

\section{Questionnaires}

Women were asked to self-complete paper-based questionnaires before (all women) and after (only women who provided samples) sample collection to assess the acceptability of collecting samples in this pilot as well as the acceptability of additional samples planned for the main study (maternal and baby urine, vaginal swabs, placental tissue and baby saliva). The pre-sample and postsample questionnaires are available in Additional files 2 and 3 , respectively.

Women were asked for their opinions about and satisfaction with the study overall as well as about the acceptability of providing each sample using a five point Likert scale ("very satisfied / comfortable" to "very unsatisfied / uncomfortable"). Women could also provide free-text comments.

\section{Data analysis}

Comparisons were made between women who did and not provide at least one sample. Comparisons were also made by sample type for women who provided at least 
one sample. For maternal stool, the denominator for these analyses included only those women who were approached to provide a stool sample $(n=85)$.

Comparisons of continuous variables, such as maternal age, were made using Student's t-tests. Categorical variables were compared using either Chi-squared or Fisher's exact tests. Means across groups were compared using one-way analysis of variance (ANOVA). All analyses were carried out using STATA version 12.0.

\section{Results}

Of 265 women approached, 171 (65\%) of women consented to participate and $113(66 \%)$ of these women gave at least one sample (Fig. 1). Seventeen women consented to participate but did not provide any samples or complete a pre-sample questionnaire.

The mean age of women who consented to participate was 34 years. Women were primarily of white ethnicity $(130 / 166,78 \%)$ and half were nulliparous $(86 / 169,51 \%)$ (Table 1). Among 168 women where mode of delivery was recorded, $57 \%$ delivered vaginally (unassisted or instrumental), nearly one third (29\%) by planned prelabour caesarean section, and $15 \%$ by intrapartum caesarean section. For 140 women who consented to participate and where information on day and time of birth were recorded, approximately half delivered outside of normal office hours (75/140, 54\%).

Women who delivered by planned pre-labour caesarean section were most likely to provide at least one sample (98\%) and those delivering by unassisted spontaneous vaginal $(50 \%)$ or ventouse were least likely $(50 \%)$ to provide a sample (Table 1). In 110 women who provided at least one sample and where information on day and time of birth were recorded, 47\% (52/110) delivered outside of normal office hours. We observed no significant difference between whether a sample was given and maternal age ( $p=0.09$, continuous variable), ethnicity $(p=0.56)$ or parity $(p=1.00)$, although we note the relatively small sample

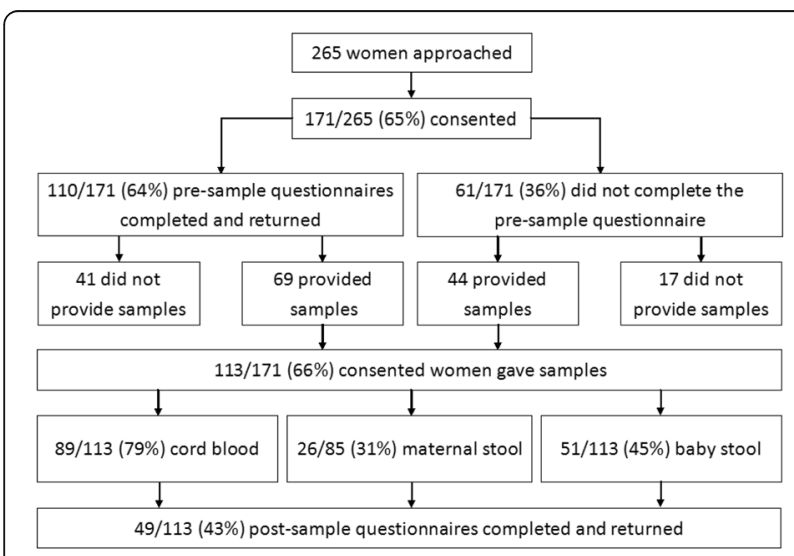

Fig. 1 Study recruitment and sample collection
Table 1 Demographic characteristics of women who consented to participate, and the proportion providing $\geq 1$ sample

\begin{tabular}{|c|c|c|c|c|c|}
\hline \multirow[b]{2}{*}{ Characteristic } & \multicolumn{2}{|l|}{ All } & \multicolumn{2}{|c|}{ Sample given } & \multirow[t]{2}{*}{$P$-value } \\
\hline & $\mathrm{n}$ & $\%$ & $n$ & $\%$ & \\
\hline \multicolumn{6}{|l|}{ Woman's ethnicity $^{a}$} \\
\hline White & 130 & 78 & 85 & 65 & \\
\hline Asian & 12 & 7 & 8 & 67 & \\
\hline Black & 14 & 8 & 11 & 79 & \\
\hline Mixed/Chinese/other & 10 & 6 & 5 & 50 & \\
\hline Total & 166 & - & 109 & - & 0.56 \\
\hline \multicolumn{6}{|l|}{ Parity $^{a}$} \\
\hline 0 & 86 & 51 & 57 & 66 & \\
\hline 1 & 63 & 37 & 42 & 67 & \\
\hline$>1$ & 20 & 12 & 14 & 70 & \\
\hline Total & 169 & - & & - & 1.00 \\
\hline \multicolumn{6}{|l|}{ Mode of delivery ${ }^{a}$} \\
\hline Vaginal & 95 & 57 & 51 & 54 & \\
\hline Unassisted & 70 & 42 & 35 & 50 & \\
\hline Forceps & 15 & 9 & 11 & 73 & \\
\hline Ventouse & 10 & 6 & 5 & 50 & \\
\hline Planned pre-labour caesarean & 48 & 29 & 47 & 98 & \\
\hline Intrapartum caesarean & 25 & 15 & 15 & 60 & \\
\hline Total & 168 & - & 113 & - & $<0.001$ \\
\hline \multicolumn{6}{|c|}{ Woman given antibiotics at any time $e^{a}$} \\
\hline Yes & 68 & & 59 & 87 & \\
\hline No & 39 & & 33 & 85 & \\
\hline Total & 107 & & 92 & - & 0.76 \\
\hline \multicolumn{6}{|l|}{ Infant given antibiotics ${ }^{a}$} \\
\hline Yes & 12 & & 11 & 92 & \\
\hline No & 117 & & 91 & 78 & \\
\hline Total & 129 & & 102 & - & 0.26 \\
\hline
\end{tabular}

an participants where data were available

size in this pilot study, which may have been underpowered to detect any difference.

Of the 113 women from whom at least one sample was collected, maternal stool was the least frequently collected sample $(26 / 85,31 \%)$, while cord blood collection was most frequently collected (89/113, 78\%). Midwives stopped collecting baby meconium after 6th May 2014 because this sample was found to contain no bacterial nucleic acid signature. Up to this date, 45 out of 70 women $(64 \%)$ had provided a meconium sample from their baby.

Whether a maternal stool sample was collected was associated with delivery mode $(p=0.01)$ (Table 2$)$. Maternal stool samples were more likely to be given by women who delivered by spontaneous vaginal delivery $(14 / 29)$ and least likely to be given by women who had planned pre-labour caesarean sections (3/31) (Table 2). 
Table 2 Association between demographic characteristics and sample collection for women who provided $\geq 1$ sample

\begin{tabular}{|c|c|c|c|c|c|c|c|c|}
\hline \multirow[t]{2}{*}{ Characteristic } & \multirow{2}{*}{$\begin{array}{l}\text { Total } \\
\text { who } \\
\text { gave } \\
\text { any } \\
\text { sample }\end{array}$} & \multicolumn{2}{|c|}{ Cord blood given } & \multirow[t]{2}{*}{$P$-value } & \multirow[b]{2}{*}{$\begin{array}{l}\text { Total } \\
\text { asked } \\
\text { to } \\
\text { provide } \\
\text { any } \\
\text { stool } \\
\text { sample }\end{array}$} & \multicolumn{2}{|c|}{ Maternal stool given } & \multirow[t]{2}{*}{$P$-value } \\
\hline & & $n$ & $\%$ & & & $n$ & $\%$ & \\
\hline \multicolumn{9}{|l|}{ Woman's ethnicity $^{a}$} \\
\hline White & 85 & 66 & 78 & & 65 & 21 & & \\
\hline Asian & 8 & 5 & 63 & & 5 & 2 & & \\
\hline Black & 11 & 10 & 91 & & 8 & 1 & & \\
\hline Mixed/Chinese/other & 5 & 5 & 100 & & 4 & 1 & & \\
\hline Total & 109 & 86 & - & 0.37 & 84 & 25 & - & 0.77 \\
\hline \multicolumn{9}{|l|}{ Parity $^{a}$} \\
\hline 0 & 57 & 44 & 77 & & 45 & 13 & & \\
\hline 1 & 42 & 32 & 76 & & 32 & 11 & & \\
\hline$>1$ & 14 & 13 & 93 & & 9 & 2 & & \\
\hline Total & 113 & 89 & - & 0.47 & 85 & 26 & - & 0.74 \\
\hline \multicolumn{9}{|l|}{ Mode of delivery ${ }^{a}$} \\
\hline \multicolumn{9}{|l|}{ Vaginal } \\
\hline Unassisted & 35 & 25 & 71 & & 29 & 14 & 48 & \\
\hline Forceps & 11 & 7 & 64 & & 9 & 4 & 44 & \\
\hline Ventouse & 5 & 3 & 60 & & 5 & 1 & 20 & \\
\hline Planned pre-labour caesarean & 47 & 42 & 89 & & 31 & 3 & 10 & \\
\hline Intrapartum caesarean & 15 & 12 & 80 & & 11 & 4 & 36 & \\
\hline Total & 113 & 89 & - & 0.09 & 85 & 26 & - & 0.01 \\
\hline
\end{tabular}

${ }^{a}$ In participants where data were available

${ }^{\mathrm{b}} \mathrm{A}$ total of 85 women were asked to provide maternal stool

We observed no evidence of an association between collection of cord blood and any of the demographic characteristics measured (Table 2). The overall mean volume of blood collected was $12.6 \mathrm{ml}$ (range: 1 to $40 \mathrm{ml}$ ), and this was not associated with either mode of delivery ( $p=0.10$ : one way ANOVA).

One hundred and ten of 171 (64\%) women who agreed to take part in the study completed a pre-sample questionnaire, including 41 women who did not provide a sample (Fig. 1). Over 95\% of women reported that they were satisfied with the explanation about the study, why samples needed to be collected, and what taking part involved (105/110, 95\%; 108/110, 98\%; and 110/110, 100\%, respectively). Women generally felt comfortable about samples being collected from their baby (Table 3). Most women $(67 \%)$ also reported being comfortable or very comfortable with giving their own stool sample (Table 3). Fewer women felt comfortable about giving a vaginal swab (53\%) (Table 3).

Forty-nine of the 113 (43\%) women who gave at least one sample returned a post-sample questionnaire. Overall satisfaction with study explanations was very similar to the pre-sample questionnaires (data not shown), and most women reported that they were comfortable with sample collection in the post-sample questionnaire (Table 3).

Free text feedback was provided by 64 women. Several women reported feeling uncomfortable about the idea of having a vaginal swab collected, which they felt might be invasive and painful. Eleven women (17\%) reported feeling uncomfortable about providing their own stool due to embarrassment and because this is not a routine procedure (in contrast to collection of urine). Seven women (11\%) raised concerns about posting baby stool samples due to the demands of caring for a newborn baby.

\section{Discussion}

This study provides evidence about biological sample collection rates from women in late pregnancy, at around the time of delivery and from their babies, and provides insight into whether or not women feel comfortable with sample collection. Overall, sample collection success was good, with 66\% (113/171) of women recruited providing 
Table 3 Pre- and post-sample responses to the question, 'Overall, how comfortable do you feel with the idea of providing the following samples?'

\begin{tabular}{llll}
\hline Sample & Pre-sample & & Post-sample \\
& $n$ & & $n \quad \%$ \\
\hline
\end{tabular}

\begin{tabular}{|c|c|c|c|c|}
\hline \multicolumn{5}{|l|}{ Maternal stool } \\
\hline Very comfortable / comfortable & 72 & 67 & 27 & 57 \\
\hline Neither & 16 & 15 & 3 & 6 \\
\hline Uncomfortable / very uncomfortable & 19 & 18 & 17 & 36 \\
\hline Total & 107 & & 47 & \\
\hline \multicolumn{5}{|l|}{ Maternal urine } \\
\hline Very comfortable / comfortable & 96 & 97 & 41 & 93 \\
\hline Neither & 2 & 2 & 2 & 5 \\
\hline Uncomfortable/Very uncomfortable & 1 & 1 & 1 & 2 \\
\hline Total & 99 & & 44 & \\
\hline \multicolumn{5}{|l|}{ Maternal vaginal swab } \\
\hline Very comfortable / comfortable & 52 & 53 & 26 & 59 \\
\hline Neither & 22 & 22 & 7 & 16 \\
\hline Uncomfortable / very uncomfortable & 24 & 25 & 11 & 25 \\
\hline Total & 98 & & 44 & \\
\hline \multicolumn{5}{|l|}{ Cord blood } \\
\hline Very comfortable / comfortable & 97 & 87 & 47 & 96 \\
\hline Neither & 7 & 7 & 2 & 4 \\
\hline Uncomfortable / very uncomfortable & 7 & 7 & 0 & 0 \\
\hline Total & 108 & & 49 & \\
\hline \multicolumn{5}{|l|}{ Stool from baby's nappy } \\
\hline Very comfortable / comfortable & 100 & 93 & 43 & 92 \\
\hline Neither & 3 & 3 & 3 & 6 \\
\hline Uncomfortable / very uncomfortable & 5 & 5 & 1 & 2 \\
\hline Total & 108 & & 47 & \\
\hline \multicolumn{5}{|l|}{ Urine from baby's nappy } \\
\hline Very comfortable / comfortable & 91 & 93 & - & - \\
\hline Neither & 4 & 4 & - & - \\
\hline Uncomfortable / very uncomfortable & 3 & 3 & - & - \\
\hline Total & 98 & & - & - \\
\hline \multicolumn{5}{|l|}{ Saliva from baby's mouth } \\
\hline Very comfortable / comfortable & 82 & 84 & - & - \\
\hline Neither & 7 & 7 & - & - \\
\hline Uncomfortable / very uncomfortable & 9 & 9 & - & - \\
\hline Total & 98 & & - & - \\
\hline
\end{tabular}

34 women responded to both questionnaires

at least one sample and most reporting that they found the study acceptable.

In general, samples were more likely to be provided when delivery was by planned pre-labour caesarean section (except maternal stool), probably because pre-labour caesarean sections were easier to plan and prepare for, as the time of delivery was known in advance. The Monday to Friday working pattern of the study's research midwives may also have impacted on sample collection success; 54\% of women who consented to participate delivered outside of normal office hours. A major constraint on sample collection may therefore have been this practical issue rather than a lack of willingness from women having vaginal deliveries. This is reflected in both the percent of those approached who consented to participate $(171 / 265,65 \%)$ and in generally high levels of acceptability for most samples in responses to pre-sample questionnaires.

The collection rate for maternal stool samples was low at $31 \%$. This may have been due in part to our deliberate selection of women who delivered by planned pre-labour caesarean section, from whom fewer stool samples were collected, probably because passing of stool during Csection delivery is unlikely. Since fewer deliveries would be by caesarean section in a population-based study [9], we anticipate that maternal stool sample collection may be higher in our main study.

It was encouraging that the proportion of women from whom cord blood was collected was high (79\%), despite the fact that cord blood banking was given precedence over our own sample collection. In the absence of cord blood banking, samples could potentially be collected from a higher number of participants, and/or larger volumes obtained.

Overall, the study and procedures were acceptable to most participants, with particularly high acceptability of collection of maternal and baby urine, baby stool and cord blood. We were surprised that the collection of vaginal swabs was perceived to be painful to collect or too invasive because such swabs have been shown to be highly acceptable in other settings such as sexual health clinics and for cervical smear screening [11, 12]. Such concerns may be alleviated by enabling self-collection and providing more information about what the procedures involve.

A limitation of this study is that it was carried out in specific late pregnancy clinics in a central London teaching hospital, and the women recruited might therefore not be representative of pregnant women in the general population. Moreover, some selection bias is possible, with those women who participated being more likely to find the study acceptable. For a small number of participants, some data items were missing. Due to the scale of this pilot we were not able to include women who needed a translator to understand the study materials. For women who consented to take part but did not provide a samples, we were unable to determine whether this was because their changed their minds or because they lacked an opportunity to provide the sample.

Our pilot found similar or somewhat better cord blood collection rates than other studies. For example, a pilot 
study for the ELFE longitudinal cohort in France reported cord blood collection for 82\% of participating mothers, which was similar to our study, [13] whereas a feasibility study in Germany reported cord blood collection for 54\% of women who consented [6]. Few gut microbiota studies report response rates or information about acceptability of stool collection, and most to date have been small in scale [14, 15]. Where reported, stool sample response rates have varied considerably between studies. For example, the Canadian CHILD study reported 3 -month and 1-year stool samples collected at home by visiting nurses were available for 33\% (422/1264) babies, [16] while stool samples collected from children aged 111 months were available for $24 \%$ of participants in the American WHEAL cohort study, [17] and a feasibility study in Germany in 751 - to 3-year-old children reported stool sample for $65.3 \%$ of participants [9]. Overall, we found few examples in the literature of dedicated studies describing the acceptability, responses rates and challenges when collecting cord blood and stool from mothers and babies around the time of birth, which emphasises the importance of the data reported here.

\section{Conclusions}

Overall, our study indicates that it is acceptable and possible to collect biological samples from pregnant women in a clinical setting using research midwives and routine National Health Service (NHS) staff, which has important implications for study design and protocol development for large-scale birth cohorts collecting biological samples around the time of birth. The major constraints to sample collection are likely to be logistical rather than related to acceptability and willingness to participate.

\section{Additional files}

Additional file 1: Baby Biome Study Collection and processing protocol: samples at birth. (PDF $2102 \mathrm{~kb}$ )

Additional file 2: Pre-birth questionnaire, pilot study questionnaire given to all women who consented to participate prior to sample collection. (PDF $368 \mathrm{~kb}$ )

Additional file 3: Post-birth questionnaire, pilot study questionnaire given to all women who gave at least one sample. (PDF 357 kb)

\section{Abbreviations}

ANOVA: Analysis of variance; NHS: National Health Service; UCLH: University College London Hospital

\section{Acknowledgements}

The authors would like to thank the study participants and staff at University College London Hospital for their time and contribution to the study.

\section{Funding}

This pilot study was funded by a Wellcome Trust Strategic Award to University College London (UCL), grant number WT101169MA. NF was funded by a National Institute for Health Research (NIHR) Academic Clinical Lectureship.

\section{Availability of data and materials}

The dataset supporting the conclusions of this article is not being made publically available to avoid potential deductive disclosure because the data are from a defined time period at one named hospital with small numbers of patients.

\section{Authors' contributions}

SRB co-wrote the study protocol, managed the data and performed all data analysis, and wrote the first draft of this article. CLT obtained funding, coconceived the study, contributed to interpretation of results and revisions of the article. $\mathrm{HD}$ and $\mathrm{CM}$ co-wrote the study protocol and collected all participant data. ET co-wrote the study protocol and contributed to revisions of the article. EMR obtained funding, co-conceived the study, contributed to study design and protocols and revisions of the article. MN and TL obtained funding, co-conceived the study and contributed to study design and protocols. AR and PB (principal investigator) obtained funding, co-conceived the study, contributed to study protocols, contributed to the analysis, interpretation of results and revisions of the article. NF obtained funding coconceived the study, supervised data collection, contributed to the analysis, interpretation of results and revising the manuscript critically for intellectual content and co-wrote study protocols. All authors read and approved the final version of the manuscript for publication.

\section{Ethics approval and consent to participate}

The study was approved by the London City and East Research Ethics Committee (REC number 12/LO/1492). Participants provided written consent to take part in the study.

Consent for publication

Not applicable.

\section{Competing interests}

The authors declare that they have no competing interests.

\section{Publisher's Note}

Springer Nature remains neutral with regard to jurisdictional claims in published maps and institutional affiliations.

\section{Author details}

'UCL Institute of Child Health, University College London, London, UK. ${ }^{2}$ University College London Hospital, London, UK. ${ }^{3}$ Division of Infection and Immunity, University College London, London, UK. ${ }^{4}$ Faculty of Infectious and Tropical Diseases, London School of Hygiene and Tropical Medicine, London, UK. ${ }^{5}$ Wellcome Trust Sanger Institute, Cambridge, UK. ${ }^{6}$ Institute for Global Health, University College London, London, UK. IInstitute for Women's Health, University College London, London, UK. ${ }^{8}$ Institute of Applied Health Research, University of Birmingham, Birmingham, UK.

Received: 11 March 2016 Accepted: 13 December 2017

Published online: 28 December 2017

References

1. Hobcraft J. Reflections on the incorporation of biomeasures into longitudinal social surveys: an international perspective. Biodemography Soc Biol. 2009:55:252-69. 10.1080/19485560903382460.

2. Richmond RC, Al-Amin A, Smith GD, et al. Approaches for drawing causal inferences from epidemiological birth cohorts: a review. Early Hum Dev. 2014;90:769-80. 10.1016/j.earlhumdev.2014.08.023.

3. Kuh D, Pierce $M$, Adams J, et al. Cohort profile: updating the cohort profile for the MRC National Survey of health and development: a new clinic-based data collection for ageing research. Int J Epidemiol. 2011;40:e1-9. 10.1093/ ije/dyq231.

4. Palmer LJ. UK Biobank: bank on it. Lancet Lond Engl. 2007;369:1980-2. 10. 1016/S0140-3656736(07)60924-6.

5. Jackson C, Best N, Elliott P. UK biobank pilot study: stability of haematological and clinical chemistry analytes. Int J Epidemiol. 2008;37:i1622. 10.1093/ije/dym280.

6. Medical Research Council. Maximising the value of UK population cohorts MRC strategic review of the largest UK population cohort studies. 2014. https://www.mrc.ac.uk/publications/browse/maximising-the-value-of-ukpopulation-cohorts/. Accessed 8 Jun 2017. 
7. Ng JWY, Barrett LM, Wong A, et al. The role of longitudinal cohort studies in epigenetic epidemiology: challenges and opportunities. Genome Biol. 2012; 13:246. 10.1186/gb-2012-13-6-246.

8. Ernst SA, Günther K, Frambach T, et al. Prenatal recruitment of participants for a birth cohort study including cord blood collection: results of a feasibility study in Bremen, Germany. Ger Med Sci GMS E-J. 2015;13:Doc04. 10.3205/000208

9. Kirkland SA, Raina PS, Wolfson C, et al. Exploring the acceptability and feasibility of conducting a large longitudinal population-based study in Canada. Can J Aging Rev Can Vieil. 2009;28:231-42. 10.1017/ S0714980809990043.

10. Field $\mathrm{N}$, Townsend $\mathrm{CL}$, Rodger AJ, et al. Infection and immunity from a lifecourse perspective: life study enhancement. Lancet. 2013;382:S35. 10. 1016/S0140-6736(13)62460-5.

11. Braz NSDF, Lorenzi NPC, Sorpreso ICE, et al. The acceptability of vaginal smear self-collection for screening for cervical cancer: a systematic review. Clinics. 2017;72:183-7. 10.6061/clinics/2017(03)09.

12. Paudyal P, Llewellyn C, Lau J, et al. Obtaining self-samples to diagnose curable sexually transmitted infections: a systematic review of patients' experiences. PLoS One. 2015;10:e0124310. 10.1371/journal.pone.0124310

13. Oleko A, Betsou F, Sarter H, et al. A pilot study of the ELFE longitudinal cohort: feasibility and preliminary evaluation of biological collection. Biopreservation Biobanking. 2011;9:223-7. 10.1089/bio.2010.0032.

14. Chu DM, Ma J, Prince AL, et al. Maturation of the infant microbiome community structure and function across multiple body sites and in relation to mode of delivery. Nat Med. 2017:23:314-94. 26

15. Yassour $\mathrm{M}$, Vatanen $\mathrm{T}$, Siljander $\mathrm{H}$, et al. Natural history of the infant gut microbiome and impact of antibiotic treatment on bacterial strain diversity and stability. Sci Transl Med. 2016;8:343ra81. 10.1126/scitranslmed.aad0917.

16. Arrieta M-C, Stiemsma LT, Dimitriu PA, et al. Early infancy microbial and metabolic alterations affect risk of childhood asthma. Sci Transl Med. 2015;7: 307ra152. 10.1126/scitransImed.aab2271.

17. Fujimura KE, Sitarik AR, Havstad S, et al. Neonatal gut microbiota associates with childhood multisensitized atopy and T cell differentiation. Nat Med. 2016:22:1187-91. 10.1038/nm.4176.

\section{Submit your next manuscript to BioMed Central and we will help you at every step:}

- We accept pre-submission inquiries

- Our selector tool helps you to find the most relevant journal

- We provide round the clock customer support

- Convenient online submission

- Thorough peer review

- Inclusion in PubMed and all major indexing services

- Maximum visibility for your research

Submit your manuscript at www.biomedcentral.com/submit

) Biomed Central 